\title{
Correction to: Natural history of fibrodysplasia ossificans progressiva: cross- sectional analysis of annotated baseline phenotypes
}

Robert J. Pignolo ${ }^{1 *}$, Geneviève Baujat ${ }^{2}$, Matthew A. Brown ${ }^{3}$, Carmen De Cunto ${ }^{4}$, Maja Di Rocco ${ }^{5}$, Edward C. Hsiao ${ }^{6}$, Richard Keen ${ }^{7}$, Mona Al Mukaddam ${ }^{8}$, Kim-Hanh Le Quan Sang ${ }^{2}$, Amy Wilson ${ }^{9}$, Barbara White ${ }^{9}$,

Donna R. Grogan ${ }^{9}$ and Frederick S. Kaplan ${ }^{10^{*}}$

Corretion to: Orphanet J Rare Dis (2019) 14:98 https://doi.org/10.1186/s13023-019-1068-7

The original version of this article [1] unfortunately included an error to an author's name. Author Maja Di Rocco was erroneously presented as Maja DiRocco.

The correct author name has been included in the author list of this Correction article and is already updated in the original article.

For additional clarity, the Correction article also provides below an updated Table 2 .

\section{Author details}

'Department of Medicine, Mayo Clinic, Rochester, MN, USA. ²Departement de Genetique, Institut IMAGINE and Hôpital Necker-Enfants Malades, Paris, France. ${ }^{3}$ Institute of Health and Biomedical Innovation (IHBI), Translational Research Institute, Princess Alexandra Hospital, Queensland University of Technology (QUT), Queensland, Australia. ${ }^{4}$ Pediatric Rheumatology Section, Department of Pediatrics, Hospital Italiano de Buenos Aires, Buenos Aires, Argentina. ${ }^{5}$ Unit of Rare Diseases, Department of Pediatrics, Giannina Gaslini Institute, Genoa, Italy. ${ }^{6}$ Division of Endocrinology and Metabolism, the UCSF Metabolic Bone Clinic, The Institute of Human Genetics, and the UCSF Program in Craniofacial Biology, Department of Medicine, University of California-San Francisco, San Francisco, California, USA. 'Centre for Metabolic Bone Disease, Royal National Orthopaedic Hospital, Stanmore, UK.

${ }^{8}$ Departments of Medicine and Orthopaedic Surgery, The Center for Research in FOP and Related Disorders, Perelman School of Medicine, University of Pennsylvania, Philadelphia, PA, USA. ${ }^{9}$ Clementia Pharmaceuticals Inc, Newton, MA, USA. ${ }^{10}$ Departments of Orthopaedic Surgery \& Medicine, The Center for Research in FOP and Related Disorders, Perelman School of Medicine, University of Pennsylvania, Philadelphia, PA, USA.
Received: 14 May 2019 Accepted: 14 May 2019

Published online: 23 May 2019

\section{Reference}

1. Pignolo RJ, et al. Natural history of fibrodysplasia ossificans progressiva: cross-sectional analysis of annotated baseline phenotypes. Orphanet J Rare Dis. 2019;14:98. https://doi.org/10.1186/s13023-019-1068-7.

\footnotetext{
* Correspondence: pignolo.robert@mayo.edu;

frederick.kaplan@uphs.upenn.edu

'Department of Medicine, Mayo Clinic, Rochester, MN, USA

${ }^{10}$ Departments of Orthopaedic Surgery \& Medicine, The Center for Research in FOP and Related Disorders, Perelman School of Medicine, University of Pennsylvania, Philadelphia, PA, USA

Full list of author information is available at the end of the article
} 
Table 2 Demographics and Baseline Disease by Age Category

\begin{tabular}{|c|c|c|c|c|c|}
\hline & $<8$ Yrs $(\mathrm{N}=17)$ & 8 to $<15$ Yrs $(\mathrm{N}=36)$ & 15 to $<25$ Yrs $(\mathrm{N}=34)$ & $\geq 25$ to $\leq 65$ Yrs $(N=27)$ & Total $(\mathrm{N}=114)$ \\
\hline Males, n (\%) & $9(52.9)$ & $24(66.7)$ & $16(47.1)$ & $13(48.1)$ & $62(54.4)$ \\
\hline \multicolumn{6}{|l|}{ Age (years) } \\
\hline Mean \pm SD & $5.9 \pm 1.1$ & $11.4 \pm 2.1$ & $18.9 \pm 3.1$ & $31.7 \pm 6.7$ & $17.6 \pm 9.7$ \\
\hline Median (min, max) & $6.0(4,7)$ & $11.0(8,14)$ & $18.5(15,24)$ & $30.0(25,56)$ & $15.0(4,56)$ \\
\hline \multicolumn{6}{|l|}{ Age at $1^{\text {st }}$ flare-up } \\
\hline Mean $\pm \mathrm{SD}$ & $2.9 \pm 2.1$ & $4.4 \pm 3.6$ & $5.6 \pm 4.8$ & $7.1 \pm 5.0$ & $5.2 \pm 4.4$ \\
\hline Median (min, max) & $2.0(1,6)$ & $4.0(0,13)$ & $3.5(0,17)$ & $5.0(0,20)$ & $4.0(0,20)$ \\
\hline \multicolumn{6}{|l|}{ Years since last flare-up } \\
\hline Mean \pm SD & $0.7 \pm 0.9$ & $1.5 \pm 2.8$ & $1.5 \pm 1.9$ & $2.3 \pm 3.5$ & $1.6 \pm 2.6$ \\
\hline Median (min, max) & $0.3(0,3)$ & $0.5(0,14)$ & $0.7(0,7)$ & $0.9(0,15)$ & $0.5(0,15)$ \\
\hline \multicolumn{6}{|c|}{ Number of flare-ups in the past 12 months (in those with flare-ups) } \\
\hline$n$ & 13 & 25 & 21 & 17 & 76 \\
\hline Mean \pm SD & $2.9 \pm 2.6$ & $6.8 \pm 11.2$ & $2.2 \pm 1.8$ & $1.9 \pm 1.6$ & $3.8 \pm 6.9$ \\
\hline Median (min, max) & $2.0(1,10)$ & $2.0(1,40)$ & $1.0(1,8)$ & $1.0(1,7)$ & $2.0(1,40)$ \\
\hline \multicolumn{6}{|l|}{ CAJIS total score ${ }^{1}$} \\
\hline Mean \pm SD & $5.6 \pm 3.9$ & $9.2 \pm 4.6$ & $13.9 \pm 6.7$ & $16.5 \pm 7.3$ & $11.8 \pm 7.0$ \\
\hline Median (min, max) & $6.0(1,15)$ & $8.5(1,20)$ & $13.5(1,26)$ & $18.0(1,30)$ & $10.5(1,30)$ \\
\hline \multicolumn{6}{|c|}{ FOP-PFQ \% worst total score ${ }^{2}$} \\
\hline Mean \pm SD & $34.8 \pm 26.1$ & $44.4 \pm 20.2$ & $43.1 \pm 31.0$ & $58.4 \pm 35.8$ & $46.3 \pm 27.1$ \\
\hline Median (min, max) & $41.8(1,84.6)$ & $45.2(1.9,82.7)$ & $38.4(0,100)$ & $54.5(0,100)$ & $45.5(0,100)$ \\
\hline \multicolumn{6}{|c|}{ PROMIS Global Physical Health (adult) or PROMIS Global Health (pediatric, parent proxy) (T-score) ${ }^{3}$} \\
\hline Mean \pm SD & $47.6 \pm 9.4$ & $41.8 \pm 8.7$ & $44.1 \pm 9.0$ & $42.5 \pm 7.9$ & NA \\
\hline Median (min, max) & $48.3(33,24)$ & $41.7(24,57)$ & $44.9(24,68)$ & $42.3(27,54)$ & \\
\hline \multicolumn{6}{|c|}{ PROMIS Global Mental Health (adult) (T-score) ${ }^{3}$} \\
\hline Mean $\pm \mathrm{SD}$ & NA & NA & $53.6 \pm 9.9$ & $51.4 \pm 8.5$ & $52.6 \pm 9.3$ \\
\hline Median (min, max) & & & $53.3(28,68)$ & $53.3(39,68)$ & $53.3(28,68)$ \\
\hline \multicolumn{6}{|c|}{ Total body $\mathrm{HO}$ volume, excluding head $\left(\mathrm{mm}^{3}\right)$} \\
\hline Mean \pm SD & $61951 \pm 75221$ & $159303 \pm 161779$ & $380751 \pm 363142$ & $651913 \pm 674454$ & $324631 \pm 440977$ \\
\hline Median (min, max) & $21692(0,224019)$ & $130509(0,828262)$ & $258543(0,1504849)$ & $481524(48844,2833946)$ & $173536(0,2833946)$ \\
\hline \multicolumn{6}{|c|}{ Number of regions with $\mathrm{HO}^{4}$} \\
\hline Mean \pm SD & $3.1 \pm 2.6$ & $5.1 \pm 2.7$ & $7.4 \pm 3.4$ & $8.8 \pm 3.7$ & $6.4 \pm 3.7$ \\
\hline Median (min, max) & $4.0(0,7)$ & $5.0(0,14)$ & $8.0(0,13)$ & $8.5(3,14)$ & $6.0(0,14)$ \\
\hline
\end{tabular}

${ }^{1}$ CAJIS assessed range of motion across 12 joints (left and right shoulders, elbows, wrists, hips, knees, and ankles) and three body regions (jaw, cervical spine [neck], and thoraco-lumbar spine). Each noted as: $0=$ uninvolved; $1=$ partially involved; $2=$ completely ankylosed. Total scores range from 0 to 30 with higher scores indicating more severe limitations in mobility.

${ }^{2}$ FOP-PFQ used transformed scores expressed as a percentage of the worst possible score. Lower percentages indicate better functioning; higher percentages indicate worse functioning.

${ }^{3}$ Distributions standardized such that a T-score of 50 (SD of 10) represents the average for the United States general population. Higher T-scores indicate better physical/mental health.

${ }^{4}$ Fifteen possible regions (neck, lower spine/abdomen, upper spine/chest; and both shoulders, elbows, wrists, hips, knees, and ankles).

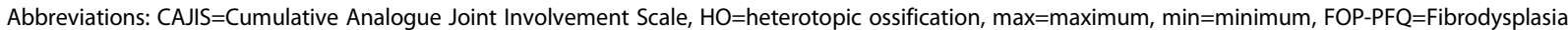
Ossificans Progressiva-Patient Function Questionnaire, NA=not applicable, PROMIS=Patient-Reported Outcome Measure Information System, SD=standard deviation, Yrs=years 